\title{
Many-body localization induced protection of topological order in a XXZ spin model
}

\author{
Yoshihito Kuno \\ Department of Physics, Graduate School of Science, Kyoto University, Kyoto 606-8502, Japan
}

(Received 10 August 2019; revised manuscript received 14 October 2019; published 27 November 2019)

\begin{abstract}
There is a counterintuitive expectation proposed by Huse et al. [Phys. Rev. B 88, 014206 (2013)] and Chandran et al. [Phys. Rev. B 89, 144201 (2014)]: Localization protects the quantum order of the ground state, even in highly excited eigenstates. In this Rapid Communication, we numerically investigate the localization protection for symmetry-protected topological (SPT) order by considering a modified XXZ spin model, related to an interacting Su-Schrieffer-Heeger (SSH) model. We systematically study how many-body localization (MBL) protects SPT for different disorder types and different forms of interactions. A certain disorder leads to the clear degeneracy of the low-lying entanglement spectrum of each excited many-body eigenstate. This fact indicates that the existence of edge modes, which is a hallmark of SPT order, is protected by the MBL in excited many-body eigenstates. In addition, we also report how the MBL-protected edge modes in excited many-body eigenstates fade away for a diagonal-type disorder.
\end{abstract}

DOI: 10.1103/PhysRevResearch.1.032026

Introduction. Many-body localization (MBL) is one of the most active topics in condensed matter physics. Theoretically, beyond the classical studies of the Anderson localization $[1,2]$, rich physical phenomena have been expected so far [3-9]. Experimentally, recent cold atom experiments have claimed to capture the ergodicity breaking dynamics of the MBL [10-13]. On the other hand, the topological state of matter is also one of the important topics in condensed matter physics. Various artificial topological states of matter have been realized in recent experimental systems [14,15] and theoretical predictions have been simulated. In this Rapid Communication, we study the interplay of MBL and the topological state. Recently, Huse et al. [16] and Chandran et al. [17] proposed a unique conjecture about the Anderson localization and MBL: A ground-state quantum order is protected even in excited many-body eigenstates, i.e., the MBL protects the ground-state quantum order. Shortly after proposing the conjecture, Kjall et al. [18] and Pekker et al. [19] have tested the MBL induced protection of ferromagnetic $\mathbf{Z}_{2-}$ symmetry breaking order by considering a one-dimensional (1D) quantum random Ising model. Bahri et al. [20] have also considered a $1 \mathrm{D}$ disordered cluster spin model as a test model and confirmed MBL induced protection of the symmetryprotected topological phase (SPT) [21,22]. This remarkable result indicates that SPT order under MBL could in principle exist out of equilibrium and, hence, cooling to detect the SPT order in real experiments is unnecessary [17,23]. Furthermore, recently, for the $1 \mathrm{D}$ disordered cluster spin model, a detailed phase diagram including the coexistence of MBL and SPT

Published by the American Physical Society under the terms of the Creative Commons Attribution 4.0 International license. Further distribution of this work must maintain attribution to the author $(s)$ and the published article's title, journal citation, and DOI. order has been investigated [24]. In addition, the relationship between the MBL and SPT phase for a time-reversal symmetric model has been extensively discussed from the perspective of the matrix product state [25,26]. However, the following issues require further investigation: (I) Does the conjecture stated in Refs. [16,17] hold universally for various condensed matter models? (II) To what extent in the whole spectrum does the mechanism of the MBL protection of quantum order work? (III) How strong does the quantum order of the ground state remain in many-body excited states? Here, we consider a modified XXZ spin model relating to the Su-Schrieffer-Heeger ( $\mathrm{SSH}$ ) model [27], which is one of the simple models exhibiting a 1D nontrivial topological insulating phase corresponding to the SPT order [28], and numerically investigate the conjecture in Refs. [16,17].

In addition, whether the SPT phase in an SSH-type model or the Affleck-Kennedy-Lieb-Tasaki (AKLT) model is protected by the MBL remains an open question [17]. Recently, a study [29] indicated that an XXZ model with a particle-hole symmetric disorder and an SSH-type coupling exhibits no protection of the SPT phase by the MBL. Here, for various conditions of disorder and interactions, we numerically evaluate the protection of the SPT phase and also the effectiveness of the arguments in Ref. [29].

Modified XXZ spin model and interacting SSH model. We consider a 1D XXZ spin model with dimerized coupling,

$$
H_{\mathrm{XXZ}}=\sum_{i}\left[J_{i}\left(S_{i}^{+} S_{i+1}^{-}+S_{i}^{-} S_{i+1}^{+}\right)+U_{i} S_{i}^{z} S_{i+1}^{z}+h_{i} S_{i}^{z}\right],
$$

where $S_{i}^{+(-)}$and $S_{i}^{z}$ are the spin-1/2 raising (lowering) operator and spin-1/2 z-component operator, respectively, and $i$ denotes the lattice site taking $i=1, \ldots, L$, where $L$ is a system size. The model parameters are shown in Fig. 1(a), for $i \in$ odd, $J_{i} \rightarrow J_{i}^{o}=J_{1}+\delta J_{i}^{o}$, for $i \in$ even, $J_{i} \rightarrow J_{i}^{e}=J_{2}+$ $\delta J_{i}^{e}$, where $\delta J_{i}^{o(e)}$ is a uniform disorder, $\left[-\delta J_{1(2)} ; \delta J_{1(2)}\right]$, and $h_{i}$ is also a uniform disorder, $[-\delta h ; \delta h]$. $U_{i}$ is regarded as 
(a)

(b)

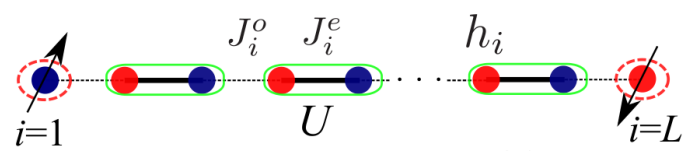

(c)
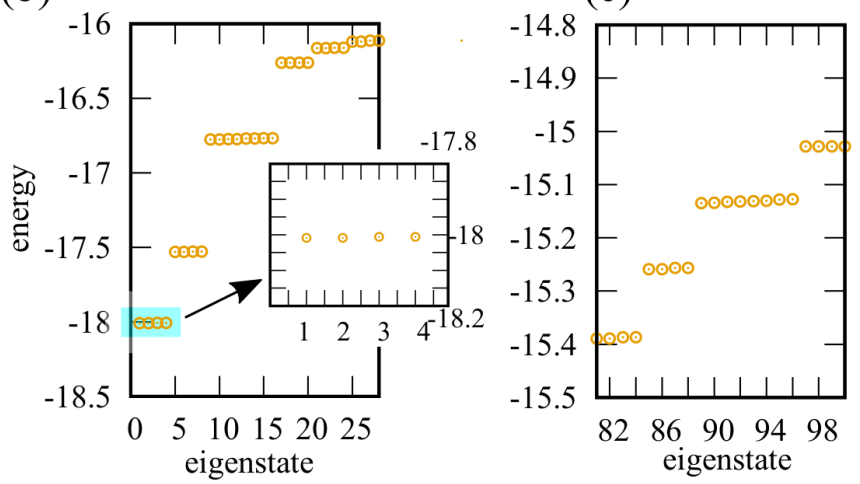

FIG. 1. (a) Modified XXZ spin model with the open boundary condition. The red-dotted circles on either end represent the edge sites of the chain. In the SPT phase $\left(J_{2}>J_{1}\right)$, the edge mode appears as a free spin- $1 / 2$ degree of freedom, corresponding to zero-energy excitation (in the thermodynamic limit). (b) Lowest to 20th energy spectrum for the $L=18$ system. (c) 81th to 100th energy spectrum with the same parameters as in (b).

an interaction in a fermion picture and here acts only on the $i \in$ even links with a strength $U$. This interaction pattern $U_{i}$ may be implemented by considering a combination of a double well and a spin-dependent optical lattice [30]. Using the Jordan-Wigner transformation, the XXZ model of $H_{\mathrm{XXZ}}$ can be mapped into an interacting SSH model [30,31] with disorder,

$$
\begin{aligned}
H_{\mathrm{SSH}}= & \sum_{i}\left[J_{i}\left(f_{i}^{\dagger} f_{i+1}+\text { H.c. }\right)\right. \\
& \left.+U_{i}\left(n_{i}-1 / 2\right)\left(n_{i+1}-1 / 2\right)+h_{i} n_{i}\right],
\end{aligned}
$$

where $f_{i}^{(\dagger)}$ is an annihilation (creation) spinless fermion and $n_{i}=f_{i}^{\dagger} f_{i}$ is a number operator. Based on this model, let us note the topological properties. In the clean limit and the $U=0$ noninteracting case, the model belongs to the BDI class in the topological classification [32,33]. At half filling, the SSH model exhibits a nontrivial topological phase. The nontrivial topological phase is known to be the SPT phase protected by the chiral symmetry. The SPT phase remains even for a finite $U_{i}[30,31,34-36]$. The model has time-reversal and particle-hole symmetry [29], then the interacting SSH model is invariant under the chiral (sublattice) transformation, $f_{i}^{\dagger} \rightarrow(-1)^{i} f_{i}, f_{i} \rightarrow(-1)^{i} f_{i}^{\dagger}$ [37]. In a disordered case, since $\delta J_{i}^{o}$ and $\delta J_{i}^{e}$ disorders do not break the chiral symmetry, the ground-state SPT phase is expected to be fairly robust to them. In contrast, if the disorder $h_{i}$ is diagonal so that the chiral symmetry is broken, the ground-state SPT becomes fragile. Since the XXZ model of $H_{\mathrm{XXZ}}$ inherits the topological properties of the SSH model of $H_{\mathrm{SSH}}$ [38], we numerically treat the XXZ model of $H_{\mathrm{XXZ}}$. In what follows, for all numerical simulations, we fix $J_{1}=0.1, J_{2}=1$, and $\delta h=0.01$ [39], except for Fig. 4.

Phenomenology: Localization protects SPT order. The studies by Huse et al. [16] and Chandran et al. [17] state that a quantum order of the ground state in an MBL system with weak interactions is protected in low-energy excited states. The low-energy excitations above the ground state are expected to be localized due to a disorder. Since very weak interacting (almost noninteracting) excitation particles are in the Anderson localization, the excitations are suppressed from extending. As a concrete example, for the 1D quantum random Ising model the ground state is ferromagnetic. The excitation is a kink (domain wall) excitation. The excitation kinks are localized owing to disorder, and therefore the ferromagnetic domains are stable. This leads to a spin-glass order even in highly excited eigenstates $[18,19]$.

The above phenomenon may apply to the model of $H_{\mathrm{Xxz}}$. For an $L=$ even clean system with $J_{1} \ll J_{2}$ and an open boundary, the ground state of the system exhibits a valencebond-solid order in the bulk, where the singlet state is formed on $J_{i}^{e}$ links. The ground state is in the SPT phase because on the left and right edge sites a free spin- $1 / 2$ degree of freedom exists [see Fig. 1(a)] corresponding to the zero-energy edge mode [40]. The edge modes induce fourfold degeneracy of the ground state. Here, let us consider the $2 J_{2}=U$ case and a single excitation on a single $J_{i}^{e}$ link. The singlet ground state on the link excites to three triplet states. The excitation may be regarded as a triplon as in the AKLT model [17]. In low-energy excited eigenstates, such triplon excitations are dominant. If we regard the triplon excitation as a quasiparticle, the quasiparticles may be localized under a finite disorder. It is expected that the localization of the quasiparticles does not affect (interact with) the edge modes, and hence the edge modes are protected by localization of the quasiparticles. This means that the SPT order of the ground state survives even in excited eigenstates. Here, under the change of $U$, disorder type, and the target sector of the excited eigenstates, it is interesting how the SPT order sustains or does not sustain.

In our $H_{\mathrm{XxZ}}$ model, we observe a certain typical signal of the protection of the SPT order by localization. Using the exact diagonalization [41-44], we numerically calculated the low-energy spectrum for the $L=18$ system with an open boundary, $U=2, \delta J_{2}=3$ [45], where edge modes are expected to appear on both sides of the edge. In the clean system, the ground state is fourfold degenerate. In Figs. 1(b) and 1(c), we plot the lowest to 28th and the 81th to 100th spectrum. We captured a fairly fourfold degeneracy of the spectrum. As observed in the inset of Fig. 1(b), the deviation is negligibly small despite the existence of the finite-size effect. Accordingly, the edge mode degeneracy seems to be protected in low-energy excited states.

Entanglement spectrum and the degeneracy. We obtained the signal of the protection of the SPT order from the spectrum degeneracy. To verify this phenomenon in detail, we calculate the entanglement spectrum (ES). Consider dividing the system into two equal halves ( $A$ and $B$ partial systems). The ES $\left\{\Lambda_{\ell}^{n}\right\}$ is defined by $\Lambda_{\ell}^{n}=-2 \log \left|\lambda_{\ell}^{n}\right|$, where each $\lambda_{\ell}^{n}$ is a singular value of the Schmidt decomposition for the $n$th eigenstate, and then the reduced density matrix of $n$th eigenstate is given by $\rho_{A}^{n}=\sum_{\ell}\left(\lambda_{\ell}^{n}\right)^{2}|\ell\rangle_{A}\left\langle\left.\ell\right|_{A}\right.$. In what follows, we assume that $\left\{\Lambda_{\ell}^{n}\right\}$ is ascending ordered for $\ell$. The degeneracy of the lowlying ES characterizes the SPT order. It corresponds to the number of edge modes [46]. For an interacting SSH model in 

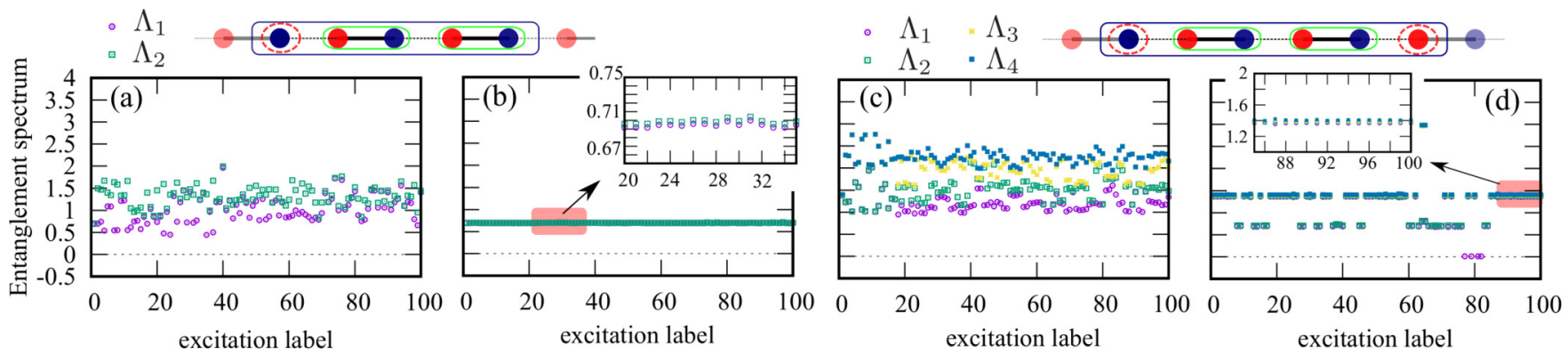

FIG. 2. Low-lying entanglement spectrum for low-energy excited eigenstates. (a) Lowest and next-lowest entanglement spectrum in the $L=14$ clean system with $U=0$, where the partial system cut has one free $1 / 2$ spin on the left edge. (b) Lowest and next-lowest entanglement spectrum in the $L=14$ disordered system with $U=2$ and $\delta J_{2}=3$. (c) Lowest to fourth entanglement spectrum in the $L=16$ clean system with $U=0$, where the partial system cut has two free $1 / 2$ spins on both the left and right edges in the SPT order. (d) Lowest and fourth entanglement spectrum in the $L=16$ disordered system with $U=2$ and $\delta J_{2}=3$.

the clean limit, the ES of the ground state has been extensively studied to characterize the SPT ground state by the ES $[31,47]$. In the clean limit, we confirmed that the SPT ground state in the SSH model of $H_{\mathrm{SSH}}$ with $J_{2}>J_{1}$ and an open boundary has two- or fourfold degeneracy of the lowest ES depending on the system size $L$.

Let us now consider the disordered system. We focus on the effects of $\delta J_{i}^{e}$ disorder in the low excited energy sector, and therefore switch off the $\delta J_{i}^{o}$ and $h_{i}$ disorders. A periodic boundary condition is considered. In the calculation of the ES, we virtually cut the system into two equal halves with the system size $L / 2$. Here, for $L / 2=$ odd, it is necessary to cut one $J_{i}^{o}$ link and one $J_{i}^{e}$ link to obtain two equal halves. If the system is in the SPT phase, the partial system has a single free spin- $1 / 2$ edge mode on either the right or left edge sites. This leads to twofold degeneracy of the lowest ES [31]. Meanwhile, for $L / 2=$ even, we cut two $J_{i}^{e}$ links to obtain two equal halves. In the SPT phase, this leads to two free spin-1/2 edge modes on both the right and left edge sites corresponding to fourfold degeneracy of the lowest ES [31]. For the $L=14$ system with $U=0$ and $\delta J_{2}=0$, we first calculated the ES by using many-body eigenstates from the ground state to the 100th excited state. The lowest and second lowest ES, $\Lambda_{1}$ and $\Lambda_{2}$, are plotted in Fig. 2(a). While $\Lambda_{1}$ and $\Lambda_{2}$ are degenerate for the ground state, $\Lambda_{1}$ and $\Lambda_{2}$ for many other excited states are not degenerate. This indicates that the SPT order vanishes in low-energy excited states except for the ground state. In contrast, we see an interacting disorder case $\delta J_{2}=3$ as shown in Fig. 2(b). This is a single-shot result for a particular $\delta J_{i}^{e}$ disorder distribution. Interestingly, we observed the proliferation of twofold degeneracy of the ES even in low-energy excited eigenstates. As shown in the inset of Fig. 2(b), the deviation of the degeneracy is negligibly small even in the finite system size. The result indicates the survival of the edge modes even in low-energy excited states due to $\delta J_{i}^{e}$ disorder. Furthermore, we also calculated the ES of the $L=16$ system for a noninteracting clean case in Fig. 2(c). The lowest to fourth lowest ES, $\Lambda_{1}, \Lambda_{2}, \Lambda_{3}$, and $\Lambda_{4}$, are plotted. As observed in Fig. 2(a), for the ground state, fourfold degeneracy from $\Lambda_{1}$ to $\Lambda_{4}$ appears, while for many other excited states fourfold degeneracy is not observed. However, as shown in Fig. 2(d), we calculated the ES for an interacting disorder case $\delta J_{2}=3$ and found the proliferation of the four- fold degeneracy of the ES even in low-energy excited states. This indicates for the $L / 2=$ even system the survival of the edge modes even in low-energy excited eigenstates. Thus, the ES calculation indicates that the $\delta J_{i}^{e}$ disorder protects the SPT order in low-energy excited eigenstates.

Further, we investigate how low-lying ES degeneracy depends on $\delta J_{2}$ and the regime of the excited energy density. To quantify the degree of the degeneracy of the low-lying ES, we define the degree of the two- or fourfold degeneracy of the ground ES, for the $L / 2=$ odd system $\Delta \Lambda_{1-2}(n) \equiv \Lambda_{2}-\Lambda_{1}$, for the $L / 2=$ even system $\Delta \Lambda_{1-4}(n) \equiv 3 \Lambda_{1}-\Lambda_{2}-\Lambda_{3}-\Lambda_{4}$, and then define the following measure,

$$
\begin{gathered}
O_{2 \mathrm{ES}}=\frac{1}{N_{s}} \sum_{n \in \mathbf{N}_{E}} \theta\left(\epsilon-\Delta \Lambda_{1-2}(n)\right), \\
O_{4 \mathrm{ES}}=\frac{1}{N_{s}} \sum_{n \in \mathbf{N}_{E}} \theta\left(\epsilon-\Delta \Lambda_{1-4}(n)\right),
\end{gathered}
$$

where $N_{s}$ is a number of eigenstates in the target regime of the spectrum denoted by $\mathbf{N}_{E}, \theta(X)$ is the Heaviside function, and $\epsilon$ represents a practical numerical criteria of the degeneracy of the ES [48]. We calculate the disorder-averaged values of $O_{2 \mathrm{ES}}$ and $O_{4 \mathrm{ES}}$ denoted by $\left\langle O_{2 \mathrm{ES}}\right\rangle$ and $\left\langle O_{4 \mathrm{ES}}\right\rangle$. In the disordered system, $\left\langle O_{2 \mathrm{ES}}\right\rangle$ and $\left\langle O_{4 \mathrm{ES}}\right\rangle$ can be regarded as an order parameter to characterize the SPT order in the excitation energy parameter space. Figure 3 shows various behaviors of $\left\langle O_{2 \mathrm{ES}}\right\rangle$ and $\left\langle O_{4 \mathrm{ES}}\right\rangle$ as varying $\delta J_{2}$ with $\delta J_{1}=0$.

In Fig. 3(a), $\left\langle O_{2 \mathrm{ES}}\right\rangle$ is of the $L=14$ system and we set $\mathbf{N}_{E}$ to $10 \%$ excited eigenstates from the ground state in all eigenstates. We found that for various $U,\left\langle O_{2 \mathrm{ES}}\right\rangle$ increases with an increase of $\delta J_{2}$, and that interestingly $\left\langle O_{2 \mathrm{ES}}\right\rangle$ saturates up to $\sim 0.6$ for large $U$. This implies that almost $60 \%$ of the excited eigenstates in the set of $\mathbf{N}_{\mathbf{E}}$ exhibit a single edge mode on the edge site. The low-energy excited eigenstates are fairly ordered in the SPT order with an increase of $\delta J_{2}$. Furthermore, for large $\delta J_{2},\left\langle O_{2 \mathrm{ES}}\right\rangle$ of the large $U$ case tends to be larger than that of small $U$. The result indicates that the interaction $U$ tends to promote the SPT order for excited eigenstates. We further calculated $\left\langle O_{2 \mathrm{ES}}\right\rangle$ with the same condition but for the middle $10 \%$ excited eigenstates in all eigenstates. Remarkably, the value of $\left\langle O_{2 \mathrm{ES}}\right\rangle$ also increases as increasing $\delta J_{2}$, and the saturated value of $\left\langle O_{2 \mathrm{ES}}\right\rangle$ is somewhat 

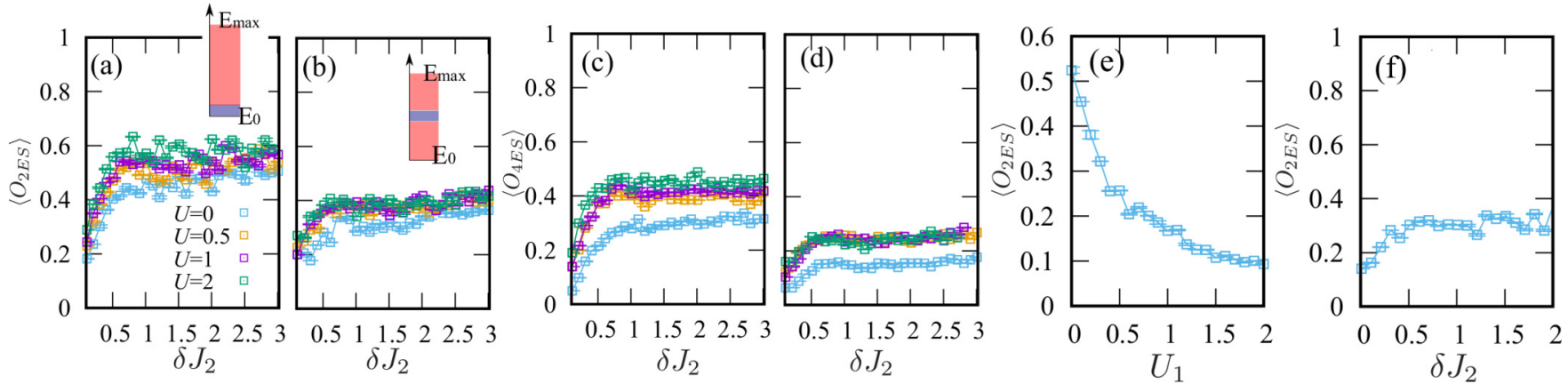

FIG. 3. The behavior of the ES degenerate order parameters with varying disorder strength $\delta J_{2}$ and an interaction form. The behavior of $\left\langle O_{2 \mathrm{ES}}\right\rangle$ in the $L=14$ system (a) for the low excited energy sector and (b) for the middle excited energy sector. The behavior of $\left\langle O_{4 \mathrm{ES}}\right\rangle$ in the $L=12$ system (c) for the low excited energy sector and (d) for the middle excited energy sector. As a whole, the interaction $U$ enhances the saturation values of $\left\langle O_{2 \mathrm{ES}}\right\rangle$ and $\left\langle O_{4 \mathrm{ES}}\right\rangle$ for large $\delta J_{2}$. (e) The effects of an odd-link interaction $U_{1}$ for the $L=14$ system with $U=2$, $\delta J_{2}=3$, and $\delta J_{1}=0$ for the low excited energy sector. (f) Modulated-disordered interaction case. $U_{i}=2 J_{i}$. The $L=14$ system with $\delta J_{2}=3$ and $\delta J_{1}=0$ for the low excited energy sector. For all cases, $10^{2}$ disorder samples were used. Error bars are small and are included in the point labels.

small compared to that in the low-energy excited sector. This indicates that the protection of the SPT order is weak in the middle excited energy sector. However, interestingly, for the high excited energy sector, the protection of the SPT order tends to be strong again, although the interaction dependence varies. See the Supplemental Material [49]. Let us turn to the disorder-dependent behavior of $\left\langle O_{4 \mathrm{ESS}}\right\rangle$ as shown in Figs. 3(c) and 3(d). The results are of $L=12$ system size. The results in Figs. 3(c) and 3(d) have the same tendency as those in Figs. 3(a) and 3(b), and therefore we expect the $L / 2=$ even system to clearly exhibit the protection of the SPT order in the low excited eigenstates. In addition, we checked the system size dependence for $\left\langle O_{2 \mathrm{ES}(4 \mathrm{ES})}\right\rangle$ (see Ref. [49]).

Other interaction conditions were also calculated. The effect of the odd-link interaction $U_{i=\text { odd }}=U_{1}$ is shown in Fig. 3(e). Here, the other parameters are $U=2$ and $\delta J_{2}=3$. As approaching a uniform interaction $U_{i}=U$ for all $i,\left\langle O_{2 \mathrm{ES}}\right\rangle$ is decreasing. The MBL protection is decreasing. The uniform interaction case tends to be close to the claim in Ref. [29]. Second, the $U_{i}=2 J_{i}$ case was also calculated as shown in Fig. 3. The parameters are the same as in Fig. 3(e). From the result, as a whole, $\left\langle O_{2 \mathrm{ES}}\right\rangle$ is smaller than those of the interacting cases in Figs. 3(a) and 3(c). Our numerics shows that the MBL protection is subtle.

Effects of diagonal disorder and MBL signal. We investigated the effects of $\delta h$ disorder. This disorder breaks the chiral symmetry in the system of $H_{\mathrm{SSH}}$. Hence, we expect that this disorder strongly affects the edge mode in the SPT order and that the SPT order tends to vanish with a certain strength of $\delta h$. Figure 4 (a) shows a typical $\delta h$ dependence of $\left\langle O_{2 \mathrm{ES}}\right\rangle$ for the $L=14$ system with $\delta J_{2}=3, \delta J_{1}=0$. For the low and middle sector of the excited energy, $\left\langle O_{2 \mathrm{ES}}\right\rangle$ decreases with an increase of $\delta h$ in various $U$. There is no clear spectrum phase transition, but a crossover towards the conventional MBL without the SPT order is observed. The protection of the SPT is fairly robust, which is almost suppressed at $\delta h \sim 1$, comparable to the $J_{2}$ energy scale. We also calculated the effects of $\delta J_{1}$ disorder and obtained a global phase diagram [49]. In addition, we investigated the stability of MBL. To characterize the MBL for each excited eigenstate, we em- ploy the average level spacing ratio $\langle r\rangle[8,50,51]$. Particularly, to obtain the clear behavior of $\langle r\rangle$, we focused on the $\sum_{i=1}^{L} S_{i}^{z}=0$ sector in the Hilbert space and on the middle excited eigenstates [50]. In the calculation of $\langle r\rangle$, consider the spectrum $\left\{E_{i}\right\}$ (in ascending order). Each level spacing in $\left\{E_{i}\right\}$ is given as $r^{k}=\left[\min \left(\delta^{(k)}, \delta^{(k+1)}\right)\right] /\left[\max \left(\delta^{(k)}, \delta^{(k+1)}\right)\right]$, where $\delta^{(k)}=E_{k+1}-E_{k}$, and then the value of $\langle r\rangle$ is obtained by averaging over disorder samples. From the value of $\langle r\rangle$, we can estimate localization in the target sector of the excited eigenstates: For the MBL, $\langle r\rangle \sim 0.386$, corresponding to the Poisson random matrix ensemble. The results of $\langle r\rangle$ are shown in Figs. 4(b) and 4(c), where we employed the $L=16$ system. Figure 4(b) shows the $\delta J_{2}$ dependence of $\langle r\rangle$ for a finite moderate $\delta h$ case $(\delta h=0.01,0.1$, and 0.2$)$ and $U=2$. For small $\delta J_{2},\langle r\rangle$ takes $\sim 0.53$ the signal of an extended state, corresponding to the Gaussian orthogonal ensemble [50,51], and with the increase of $\delta J_{2}$, the values of $\langle r\rangle$ approach $\sim 0.386$. $\delta J_{2}$ disorder induces MBL, i.e., MBL is exhibited
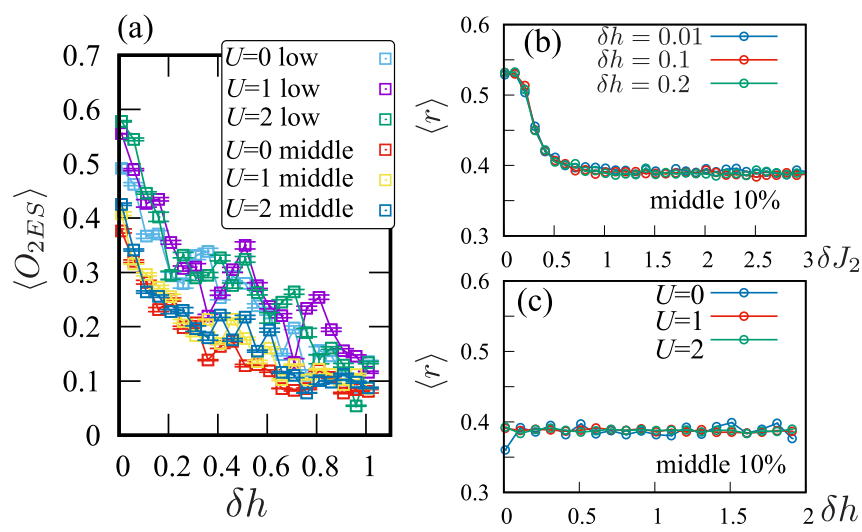

FIG. 4. (a) $\delta h$ dependence of $\left\langle O_{2 \mathrm{ES}}\right\rangle$ for the $L=14$ system with $J_{1}=0.1$ and $J_{2}=1$. Error bars are small and are included in the point labels. (b) $\delta J_{2}$ dependence of the average gap ratio $\langle r\rangle$ for the middle excited energy sector. (c) $\delta h$ dependence of the average gap ratio $\langle r\rangle$ for the middle excited energy sector. For all results, we averaged over $10^{2}$ disorder samples. 
when the eigenstates turn into the SPT order as increasing $\delta J_{2}$ from zero [52]. Figure 4(c) displays the $\delta h$ dependence of $\langle r\rangle$ with $\delta J_{2}=3$. Even in varying $\delta h$ where the SPT order is suppressed in excited eigenstates, the MBL signal $\langle r\rangle \sim 0.386$ remains. This indicates that, during the crossover behavior in Fig. 4(a), the MBL sustains.

Conclusion. For a simple modified XXZ model, we numerically investigated the MBL protection of the SPT phase. We found that for a certain interaction pattern, the MBL clearly protects the SPT order in many-body excited eigenstates. However, a uniform interaction situation and the diagonal disorder weaken the tendency of the MBL protection. Although the limitation in this work is the small system sizes accessible to exact diagonalization, our results indicate that the SPT order in the XXZ model possibly appears even in high temperatures or out of equilibrium.

Acknowledgments. Y.K. acknowledges the support of the Grant-in-Aid for JSPS Fellows (No. 17J00486). Y.K. is very grateful to Jakub Zakrzewski for his critical reading of the manuscript and for providing numerous helpful suggestions that improved the draft, and thanks an anonymous referee for valuable suggestions.
[1] P. W. Anderson, Phys. Rev. 109, 1492 (1958).

[2] A. Lagendijk, B. Van Tiggelen, and D. S. Wiersma, Phys. Today 62(8), 24 (2009).

[3] R. Nandkishore and D. A. Huse, Annu. Rev. Condens. Matter Phys. 6, 15 (2015).

[4] D. M. Basko, I. L. Aleiner, and B. L. Altshuler, Ann. Phys. 321, 1126 (2006).

[5] D. A. Abanin and Z. Papic, Ann. Phys. 529, 1700169 (2017).

[6] F. Alet and N. Laflorencie, C. R. Phys. 19, 498 (2018).

[7] D. A. Abanin, E. Altman, I. Bloch, and M. Serbyn, Rev. Mod. Phys. 91, 021001 (2019).

[8] V. Oganesyan and D. A. Huse, Phys. Rev. B 75, 155111 (2007).

[9] S. Iyer, V. Oganesyan, G. Refael, and D. A. Huse, Phys. Rev. B 87, 134202 (2013).

[10] M. Schreiber, S. S. Hodgman, P. Bordia, H. P. Luschen, M. H. Fischer, R. Vosk, E. Altman, U. Schneider, and I. Bloch, Science 349, 842 (2015).

[11] J.-Y. Choi, S. Hild, J. Zeiher, P. Schaus, A. Rubio-Abadal, T. Yefsah, V. Khemani, D. A. Huse, I. Bloch, and C. Gross, Science 352, 1547 (2016).

[12] A. Lukin, M. Rispoli, R. Schittko, M. E. Tai, A. M. Kaufman, S. Choi, V. Khemani, J. Léonard, and M. Greiner, Science 364, 256 (2019).

[13] M. Rispoli, A. Lukin, R. Schittko, S. Kim, M. E. Tai, J. Léonard, and M. Greiner, Nature (London) 573, 385 (2019).

[14] N. R. Cooper, J. Dalibard, and I. B. Spielman, Rev. Mod. Phys. 91, 015005 (2019).

[15] T. Ozawa, H. M. Price, A. Amo, N. Goldman, M. Hafezi, L. Lu, M. C. Rechtsman, D. Schuster, J. Simon, O. Zilberberg, and I. Carusotto, Rev. Mod. Phys. 91, 015006 (2019).

[16] D. A. Huse, R. Nandkishore, V. Oganesyan, A. Pal, and S. L. Sondhi, Phys. Rev. B 88, 014206 (2013).

[17] A. Chandran, V. Khemani, C. R. Laumann, and S. L. Sondhi, Phys. Rev. B 89, 144201 (2014).

[18] J. A. Kjall, J. H. Bardarson, and F. Pollmann, Phys. Rev. Lett. 113, 107204 (2014)

[19] D. Pekker, G. Refael, E. Altman, E. Demler, and V. Oganesyan, Phys. Rev. X 4, 011052 (2014).

[20] Y. Bahri, R. Vosk, E. Altman, and A. Vishwanath, Nat. Commun. 6, 7341 (2015).

[21] F. Pollmann, A. M. Turner, E. Berg, and M. Oshikawa, Phys. Rev. B 81, 064439 (2010).

[22] F. Pollmann, E. Berg, A. M. Turner, and M. Oshikawa, Phys. Rev. B 85, 075125 (2012).
[23] S. A. Parameswaran and R. Vasseur, Rep. Prog. Phys. 81, 082501 (2018)

[24] K. S. C. Decker, D. M. Kennes, J. Eisert, and C. Karrasch, arXiv:1902.02259.

[25] T. B. Wahl, Phys. Rev. B 98, 054204 (2018).

[26] A. Chan and T. B. Wahl, arXiv:1808.05656.

[27] W. P. Su, J. R. Schrieffer, and A. J. Heeger, Phys. Rev. Lett. 42, 1698 (1979).

[28] J. K. Asbóth, L. Oroszlány, and A. Pályi, A Short Course on Topological Insulators: Band Structure and Edge States in One and Two Dimensions, Lecture Notes in Physics Vol. 919 (Springer, New York, 2016).

[29] R. Vasseur, A. J. Friedman, S. A. Parameswaran, and A. C. Potter, Phys. Rev. B 93, 134207 (2016).

[30] Y. Kuno, Phys. Rev. B 99, 064105 (2019).

[31] J. Sirker, M. Maiti, N. P. Konstantinidis, and N. Sedlmayr, J. Stat. Mech. (2014) P10032.

[32] A. P. Schnyder, S. Ryu, A. Furusaki, and A. W. W. Ludwig, Phys. Rev. B 78, 195125 (2008).

[33] A. Kitaev, in Advances in Theoretical Physics: Landau Memorial Conference, edited by V. Lebedev and M. Feigel'man, AIP Conf. Proc. No. 1134 (AIP, Melville, NY, 2009), p. 22.

[34] M. Yahyavi, L. Saleem, and B. Hetenyi, J. Phys.: Condens. Matter 30, 445602 (2018).

[35] B. Hetényi and B. Dóra, Phys. Rev. B 99, 085126 (2019).

[36] M. Weber, F. P. Toldin, and M. Hohenadler, arXiv:1905.05218.

[37] S. R. Manmana, A. M. Essin, R. M. Noack, and V. Gurarie, Phys. Rev. B 86, 205119 (2012).

[38] M. P. Estarellas, I. D'Amico, and T. P. Spiller, Sci. Rep. 7, 42904 (2017)

[39] In our numerics, if we set $\delta h=0$, degeneracy in the spectrum is proliferated. This may originate from the symmetries of the topological model and the nonconservation of the total spin- $z$ component in the system. Such a degeneracy can lead to difficulty in evaluating the degeneracy of the entanglement spectrum. Once we inject small but finite $\delta h$, the degree of the degeneracy is expected to be lessened.

[40] In the thermodynamic limit, the edge mode exhibits exact zeroenergy excitation. For a finite-size case, the energy excitation is exponentially small with the system size $L$.

[41] M. Noack and S. R. Manmana, in Lectures on the Physics of Highly Correlated Electron Sysytems IX: Ninth Training Course in the Physics of Correlated Electron Systems and High-T 
Superconductors, edited by A. Avella and F. Mancini, AIP Conf. Proc. Vol. 789 (AIP, Melville, NY, 2005), pp. 93-163.

[42] M. Zhang and R. X. Dong, Eur. J. Phys. 31, 591 (2010).

[43] P. Prelovsek and J. Bonca, Strongly Correlated Systems: Numerical Methods, Springer Series in Solid-State Sciences Vol. 176 (Springer, Berlin, 2013).

[44] D. Raventos, T. Gras, M. Lewenstein, and B. Julia-Diaz, J. Phys. B 50, 113001 (2017).

[45] In all numerical simulation except for Figs. 4(b) and 4(c), the Hilbert space includes all spin- $z$ component sectors. The total spin- $z$ component $S_{\text {total }}^{z}=\sum_{i} S_{i}^{z}$ is not conserved.

[46] L. Fidkowski, Phys. Rev. Lett. 104, 130502 (2010).

[47] B. T. Ye, L. Z. Mu, and H. Fan, Phys. Rev. B 94, 165167 (2016).

[48] As shown in the insets of Figs. 2(b) and 2(d), since the deviation of the degeneracy coming from finite-size effects is negligibly small, we set $\epsilon=0.1$ for the calculation of $\left\langle O_{2 \mathrm{ES}}\right\rangle$ and $\epsilon=0.2$ for the calculation of $\left\langle O_{4 \mathrm{ES}}\right\rangle$.

[49] See Supplemental Material at http://link.aps.org/supplemental/ 10.1103/PhysRevResearch.1.032026 for degeneracy of the ES in high excited eigenstates, finite system size effects and $\delta J_{2}$ and $\delta h$ disorder phase diagram.

[50] D. J. Luitz, N. Laflorencie, and F. Alet, Phys. Rev. B 91, 081103(R) (2015).

[51] J. Janarek, D. Delande, and J. Zakrzewski, Phys. Rev. B 97, 155133 (2018).

[52] For the $\delta h=0$ case, $\langle r\rangle$ indicated is less than 0.386 . This comes from the fact that the level spacing ratio is difficult to capture the signal of the MBL when the high degeneracy of the entire spectrum of the model of $H_{\mathrm{XXZ}}$, without $h_{i}$ disorder remains. However, we expect since the very small $\delta h=0.01$ case clearly indicates the MBL signal and therefore even for the limit $\delta h \rightarrow 0$, the system with only $\delta J_{2}$ disorder is in the MBL. 